ఠ

\title{
miR-342-3p suppresses hepatocellular carcinoma proliferation through inhibition of IGF-IR- mediated Warburg effect
}

This article was published in the following Dove Press journal:

OncoTargets and Therapy

\author{
Wenpeng Liu ${ }^{1, *}$ \\ Lei Kang ${ }^{2, *}$ \\ Juqiang $\mathrm{Han}^{3}$ \\ Yadong Wang' \\ Chuan Shen' \\ Zhifeng Yan ${ }^{4}$ \\ Yanhong Tai ${ }^{5}$ \\ Caiyan Zhao'
}

'Department of Infectious Diseases, Third Affiliated Hospital of Hebei Medical University, Shijiazhuang, China; ${ }^{2}$ Department of Nuclear Medicine, Peking University First Hospital, Beijing, China; ${ }^{3}$ nstitute of Liver Disease, Beijing Military General Hospital, Beijing, China; ${ }^{4}$ Department of Gynecology and Obstetrics, PLA General Hospital, Beijing, China; ${ }^{5}$ Department of Pathology, Hospital of PLA, Beijing, China

*These authors contributed equally to this work
Background: Insulin-like growth factor-1 receptor (IGF-1R) is a well-studied oncogenic factor that promotes cell proliferation and energy metabolism and is overexpressed in numerous cancers including hepatocellular carcinoma (HCC). Aerobic glycolysis is a hallmark of cancer, and drugs targeting its regulators, including IGF-1R, are being developed. However, the mechanisms of IGF-1R inhibition and the physiological significance of the IGF-1R inhibitors in cancer cells are unclear. Materials and methods: Cell proliferation was evaluated by cell counting Kit- 8 and colony formation assay. Western blot and real-time PCR were accordingly used to detect the relevant proteins, miRNA and gene expression. Luciferase reporter assays were used to illustrate the interaction between miR-342-3p and IGF-1R. The effect of miR-342-3p on glycolysis was determined by glucose uptake, ATP concentration, lactate generation, extracellular acidification rate and oxygen consumption rate assays. In vivo, subcutaneous tumor formation assay and PET were performed in nude mice.

Results: In this study, we demonstrate that by directly targeting the 3'-UTR (3'-untranslated regions) of IGF-1R, microRNA-342-3p (miR-342-3p) suppresses IGF-1R-mediated PI3K/AKT/ GLUT1 signaling pathway both in vitro and in vivo. Through suppression of IGF-1R, miR342-3p dampens glycolysis by decreasing glucose uptake, lactate generation, ATP production, and extracellular acidification rate (ECAR), and increasing oxygen consumption rate (OCR) in hepatoma cells. Importantly, glycolysis regulated by miR-342-3p is critical for its regulating HCC growth both in vitro and in vivo.

Conclusion: Our findings provide clues regarding the role of miR-342-3p as a tumor suppressor in liver cancer mainly through the inhibition of IGF-1R. Targeting IGF-1R by miR-342-3p could be a potential therapeutic strategy in liver cancer.

Keywords: miR-342-3p, IGF-1R, liver cancer, glycolysis, metabolism, PET

\section{Introduction}

Hepatocellular carcinoma (HCC) is among the most common and lethal cancers worldwide, briefly, standing for the third leading cause of cancer death and the fifth prevalent human cancer. ${ }^{1}$ Despite much progress made to improve the therapeutic options, ${ }^{2,3}$ the long-term prognosis of HCC patients still remains poor, with a 5-year survival rate of approximately $30 \% .^{4-7}$ Therefore, further understanding of the underlying molecular mechanisms of HCC tumorigenesis is crucial and urgent.

The Warburg effect (aerobic glycolysis) is a typical feature of cancer cells, exhibiting abnormal metabolism characterized by high glycolysis even in the presence of ample oxygen. ${ }^{8,9}$ Now, the Warburg effect has been widely recognized as a hallmark of cancer which can facilitate tumor growth with enhanced glucose uptake and lactate production. 
Therefore, the understanding of the control of this process is crucial to identify potential targets for HCC therapy.

miRNAs are a group of endogenous, small noncoding RNAs interacting preferentially with the $3^{\prime}$-untranslated regions ( $3^{\prime}$-UTRs) of target mRNAs, negatively regulating gene expression post-transcriptionally. ${ }^{10,11}$ It has been demonstrated that miRNAs play vital roles in various cancers, and some miRNAs may be crucial for diagnosis, therapy and prognosis in human cancer. ${ }^{12,13}$ In previous work, researchers have identified miR-342-3p as an important cancer-related miRNA, including colon cancer, cervical cancer, gallbladder cancer and osteosarcoma. ${ }^{14-19}$ Given that miR-342-3p plays an important suppressive role in cancer, we aimed to determine whether miR-342-3p regulates the Warburg effect in HCC.

In the current study, we demonstrate that miR-342-3p suppresses aerobic glycolysis and cells proliferation through targeting IGF-1R-mediated PI3K/AKT/GLUT1 signaling pathway in liver cancer. Thus, activation of miRNA-342-3p may be a novel, promising way to treat HCC.

\section{Materials and methods}

\section{Cell lines, RNA oligonucleotides, reagents} Human liver cancer cell lines HepG2 and MHCC97H, and human embryonic kidney cell line HEK293T were purchased from the American Type Culture Collection (Manassas, VA, USA). Wild-type and mutated miR-342-3p supposed targets on IGF-1R 3'-UTR were cloned into pmir-GLO dual-luciferase miRNA target expression vector (Promega Corporation, Fitchburg, WI, USA). IGF-1R siRNA was purchased from GenePharma (Shanghai, China). Anti-IGF-1R, anti-pIGF-1R (Tyr1135) and anti-GLUT1 antibodies were purchased from Cell Signalling Technology (Danvers, MA, USA). Anti-AKT, anti-pAKT and anti- $\beta$-actin antibodies were purchased from Santa Cruz Biotechnology Inc. (Dallas, TX, USA). miR-342-3p mimics and miR-342-3p inhibitor were purchased from GenePharma.

\section{Cell growth and colony formation assays}

Cell growth was evaluated by a cell counting kit-8 (CCK-8) following the manufacturer's protocols (Dojindo, Tokyo, Japan). For colony formation assay, transfected cells were seeded in $35 \mathrm{~mm}$ plates at a density of 3,000 cells per well. Two weeks later, colonies were fixed with $4 \%$ paraformaldehyde and stained with $1 \%$ crystal violet for $30 \mathrm{~min}$. The number of colonies with diameters of more than $1.5 \mathrm{~mm}$ was counted.

\section{miRNA extraction and quantitative real- time PCR (qRT-PCR)}

Total RNA containing miRNA was extracted from cultured cells using a miRcute miRNA isolation kit (Tiangen, Beijing,
China). Target miRNA was reverse transcribed to cDNA with the miRcute miRNA First-Strand cDNA Synthesis Kit (Tiangen). The expression level of miRNA was identified by a miScript SYBR Green PCR Kit (Qiagen NV, Venlo, the Netherlands) and performed on the CFX96 system (BioRad Laboratories Inc., Hercules, CA, USA). miR-342-3p expression level was assessed by qRT-PCR with the following primers: 5'-GGGTCTCACACAGAAATCGC-3' (forward), and 5'-CAGTGCGTGTCGTGGAGT-3' (reverse). The control primers (U6) were 5'-CGCGCTTC GGCA GCACA T A T A C T - 3' (forward) and 5'-ACGCTTCACGAATTTGCGTGTC-3' (reverse). IGF-1R mRNA expression was determined by qRT-PCR with the following primers: 5'-GAGGAGATGGAGCCTGGCTTC-3' (forward), and 5'-GTGTCTGTCGGGCAGTGGCAG-3' (reverse). The control primers for $\beta$-actin were $5^{\prime}$-ATCAC CATTGGCAATGAGCG-3' (forward) and 5'-TTGA AGGTAGTTTCGTGGAT-3' (reverse). Normalized to the corresponding control, the relative quantification value of the target was computed by the comparative cycle threshold methods.

\section{Luciferase reporter assay}

Cells seeded in 24-well plates at $1 \times 10^{5}$ cells per well were cotransfected with luciferase reporters, either wild-type or mutant IGF-1R 3'-UTR, in combination with miR-342-3p mimics or negative control for miRNA mimics using Lipofectamine 2000. Forty-eight hours later, cells were harvested and analyzed for luciferase and $\beta$-galactosidase activities according to the manufacture's instruction (Promega).

\section{Glucose uptake, lactate, and ATP assays}

Glucose Uptake Colorimetric Assay kit, Lactate Assay Kit II, and ATP Colorimetric Assay kit were made use of evaluating glucose uptake, lactate production and ATP generation respectively, according to the manufacturer's instructions (BioVision, Milpitas, CA, USA). For glucose uptake colorimetric assay, cells were seeded at $1 \times 10^{4}$ per well in a 96-well plate. The cells were starved for glucose by preincubating with $100 \mu \mathrm{L}$ Krebs-Ringer-Phosphate-HEPES buffer containing $2 \%$ BSA for $40 \mathrm{~min}$. Ten microliters of $10 \mathrm{mM}$ 2-DG was added, then the cells were incubated for $20 \mathrm{~min}$. For lactate and ATP assays, $5 \times 10^{5}$ cells were homogenized in $50 \mu \mathrm{L}$ corresponding assay buffer provided by the kits. Samples were centrifuged at $4{ }^{\circ} \mathrm{C}$, and the soluble fraction was assayed.

\section{Extracellular acidification and oxygen consumption rate assays}

The extracellular acidification rate (ECAR) and cellular oxygen consumption rate (OCR) were determined using the Seahorse 
XFe 96 Extracellular Flux Analyzer (Seahorse Bioscience, North Billerica, MA, USA). Experiments were performed according to the manufacturer's instructions. ECAR and OCR were measured using Seahorse XF Glycolysis Stress Test Kit and Seahorse XF Cell Mito Stress Test Kit, respectively. Briefly, cells were seeded in a Seahorse XF 96 cell culture microplate at a density of $1 \times 10^{4}$ per well. For ECAR, after baseline measurements, glucose, the oxidative phosphorylation inhibitor oligomycin, and the glycolytic inhibitor 2-DG were sequentially injected into each well at indicated time points; and for OCR, oligomycin, the reversible inhibitor of oxidative phosphorylation FCCP ( $p$-trifluoromethoxy carbonyl cyanide phenylhydrazone), and the mitochondrial complex I inhibitor rotenone plus the mitochondrial complex III inhibitor antimycin A (Rote/AA) were sequentially injected. Data were computed by Seahorse XF-96 Wave software. ECAR is shown in $\mathrm{mpH} / \mathrm{min}$ and OCR in $\mathrm{pmol} / \mathrm{min}$.

\section{In vivo small-animal PET imaging and lactate assays}

The animal study was approved and monitored by the Ethics Committees of the Third Affiliated Hospital of Hebei Medical University (Shijiazhuang, China). All experiments were performed in accordance with the guidelines approved by Institutes of Health for the Care and Use of Laboratory Animals at the Third Affiliated Hospital of Hebei Medical University. For in vivo tumor assay, a total of $1 \times 10^{7} \mathrm{HepG} 2$ cells with stable expression of $\mathrm{pCDH}$ or $\mathrm{pCDH}-\mathrm{miR}-342$ subcutaneously inoculated into the hind flank of nude mice (6-week-old male nude mice), respectively. All mice were kept for about 60 days until imaged by small-animal positron emission tomography (PET) imaging. An animal PET scanner (Philips Corp., Amsterdam, Netherlands) was used for performing the PET of tumor-bearing mice. After they were intraperitoneally anesthetized by pentobarbital (100 mg/kg; Sinopharm Chemical Reagent, Beijing, China), mice were injected intravenously with $3.7 \mathrm{MBq}(100 \mu \mathrm{Ci})$ of $18 \mathrm{~F}$ radiolabeled fluorodeoxyglucose ( $\left.{ }^{18} \mathrm{~F}-\mathrm{FDG}\right)$. Five-minute emission scans were implemented to obtain attenuation correction data in the prone position at $60 \mathrm{~min}$ after injection. The radioactivity of tumors was measured using a $\mathrm{NaI}(\mathrm{Tl})$ well counter (China Atom Corp., Beijing, China). Themicewere sacrificedabout60 days after tumor cell inoculation, and tumor tissues were excised for lactate production and protein levels study. Tissues were homogenized in the lactate assay buffer. Insoluble materials were removed after centrifuge. The soluble fraction was assayed directly according to the Lactate Assay Kit II manufacturer's instructions (BioVision).

\section{Statistical analysis}

All the experiments were performed in triplicate and repeated 3 times in vitro. Statistical significance in cell proliferation, glucose uptake, lactate, ATP, ECAR and OCR, as well as luciferase reporter assays was determined by two-tailed Student's $t$-test. The statistical analyses were computed by the SPSS 17.0 statistical software package. $P$-values of less than 0.05 were considered statistically significant.

\section{Results}

\section{Aerobic glycolysis is critical for regulating hepatoma cell proliferation by miR-342-3p}

Since aerobic glycolysis plays a crucial role in cancer progression and miR-342-3p acts as a suppressor gene, we investigated whether aerobic glycolysis plays a role in miR-342-3p mediated suppression of hepatoma cell proliferation. Indeed, 2-deoxy-D-glucose (2-DG), the glycolytic inhibitor, weakened the ability of miR-342-3p mimics to inhibit proliferation in HepG2 and MHCC97H hepatoma cells (Figure 1A and $\mathrm{B})$. Colony formation assays proved similar trends to that of growth curves mentioned above (Figure 1C and D). These data collectively show that aerobic glycolysis could take part in the regulation of cell proliferation by miR-342-3p.

\section{Identification of IGF-IR as a direct target of miR-342-3p}

To explore the relationship between miR-342-3p and aerobic glycolysis, we searched for the potential target genes of miR-342-3p using publicly available databases (TargetScan and miRanda). Multiple genes were predicted as the potential miR-342-3p targets, from which we picked out those reported to associate with glycolysis (Figure S1). Next, we performed Western blot analysis to confirm the potential targets in human kidney embryonic HEK293T cells. As previously reported, ${ }^{27}$ overexpression of miR-342-3p mimics inhibited the E2F1 expression (Figure S1). Moreover, miR-342-3p repressed the expression of IGF-1R, a key glycolysis, but not ENO1 (alpha-enolase), another enzyme involved in glycolysis. Therefore, we chose IGF-1R for further study.

IGF-1R is well known to activate intracellular AKT signaling pathway, which subsequently upregulate the expression of GLUT1 on plasma membrane and then enhance glucose metabolism in cancer cells. ${ }^{20,21}$ Therefore, we tested if miR342-3p influenced the process mentioned above. As expected, overexpression of miR-342-3p mimics suppressed the levels of IGF-1R expression, the phosphorylation form of AKT and the GLUT1 expression in HepG2 and MHCC97H cells (Figure 2A). On the contrary, anti-miR-342-3p facilitated IGF-1R expression, and increased that of phosphorylation form of AKT and the expression level of GLUT1 (Figure 2B). To determine how miR-342-3p influenced the expression of IGF-1R, we detected the expression of IGF-1R mRNA after transfection of miR-342-3p mimics or miR-342-3p inhibitor 
A
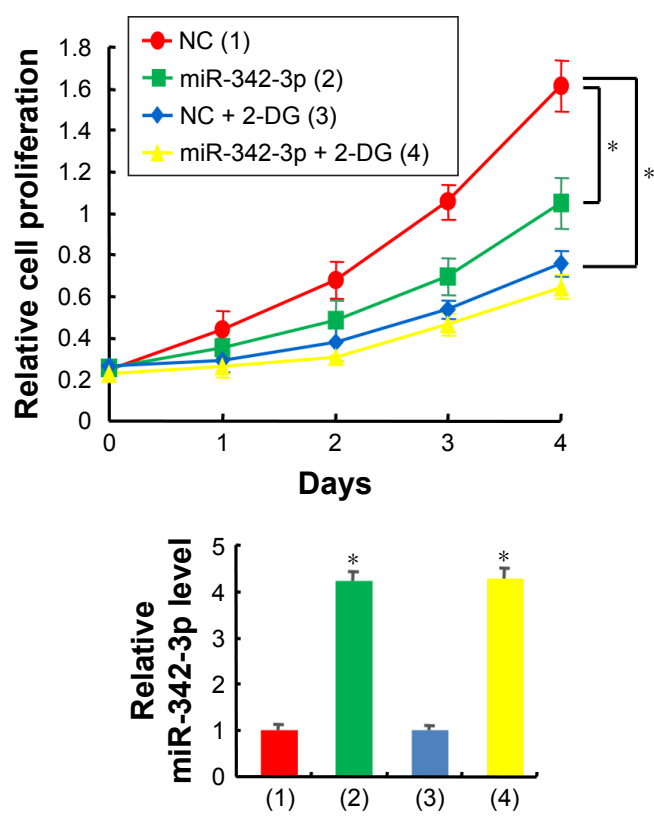

B
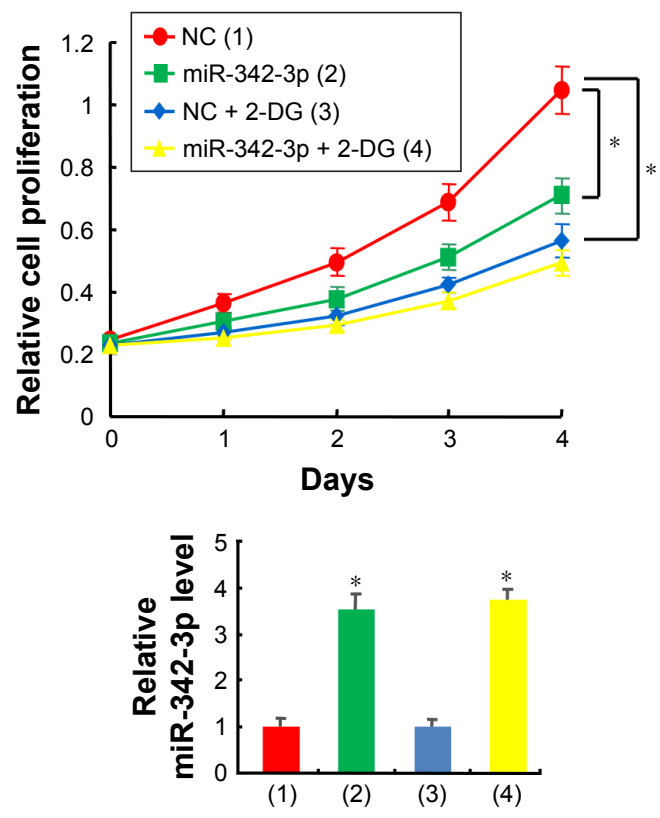

NC (1) $\quad$ miR-342-3p (2) $\quad$ NC + 2-DG (3) miR-342-3p + 2-DG (4)

C
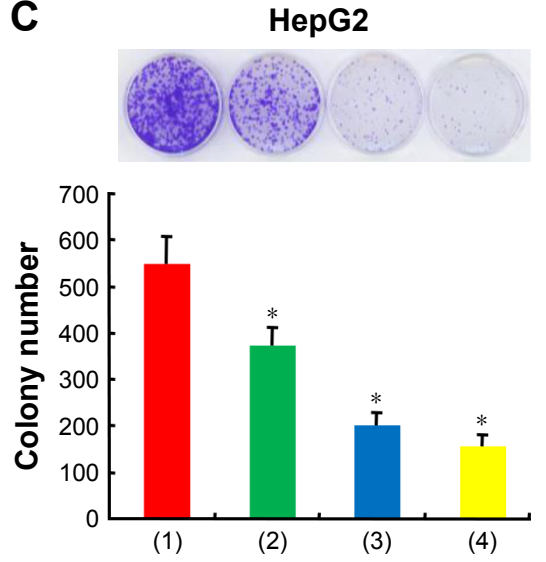

D

MHCC97H
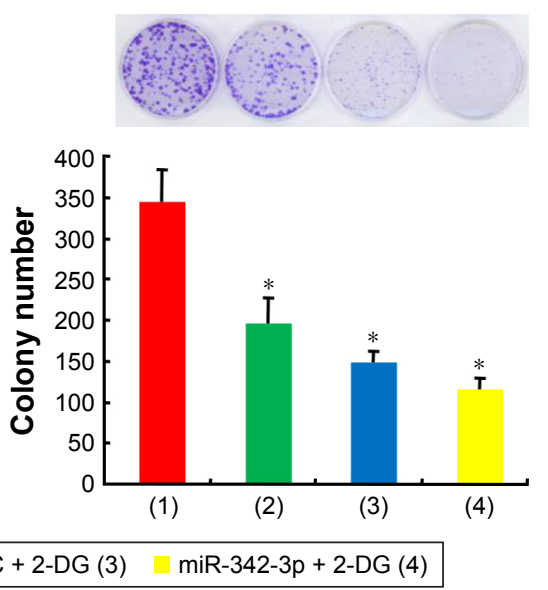

Figure I Aerobic glycolysis is involved in regulating hepatoma cell proliferation by miR-342-3p.

Notes: (A, B) The proliferation curve of HepG2 and MHCC97H hepatoma cells transfected with miR-342-3p mimics or non-specific control for miRNA mimics (NC) and treated with $2.5 \mathrm{mM}$ 2-DG as indicated. qRT-PCR analyzation suggests miR-342-3p expression level. (C, D) Colony formation assays of HepG2 and MHCC97H cells treated and analyzed as in $(\mathbf{A}$ and $\mathbf{B})$. $* P<0.01$.

Abbreviation: NC, negative control.

into HepG2 and MHCC97H cells. The levels of IGF-1R mRNA were down-regulated upon miR-342-3p overexpression, whereas the levels of IGF-1R mRNA were upregulated upon miR-342-3p inhibition (Figure 2C and D).

To further identify whether the negative regulation of miR342-3p on IGF-1R expression were mediated through binding of IGF-1R directly, we transfected HepG2 and MHCC97H cells with wild-type IGF-1R 3'-UTR or mutated IGF-1R $3^{\prime}$-UTR luciferase reporter and miR-342-3p. miR-342-3p reduced the wild-type IGF-1R 3'-UTR reporter activity, but not the luciferase activity of the reporter in which the binding sites for miR-342-3p were mutated (Figure 2E). In short, these results reveal that miR-342-3p inhibits IGF-1R expression by targeting its 3'-UTR directly in hepatoma cells.

\section{miR-342-3p suppresses cell proliferation} and glycolysis mainly through inhibition of IGF-IR expression in hepatoma cells

miR-342-3p has been shown to inhibit hepatoma cell proliferation. ${ }^{22}$ Thus, we tested if these functions mediated 
A

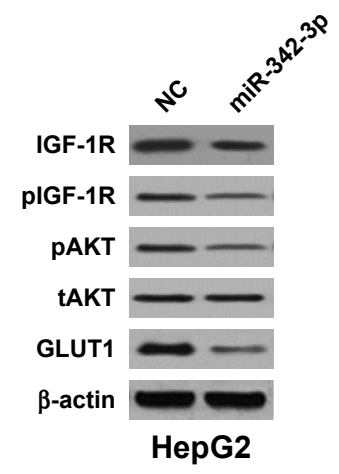

B
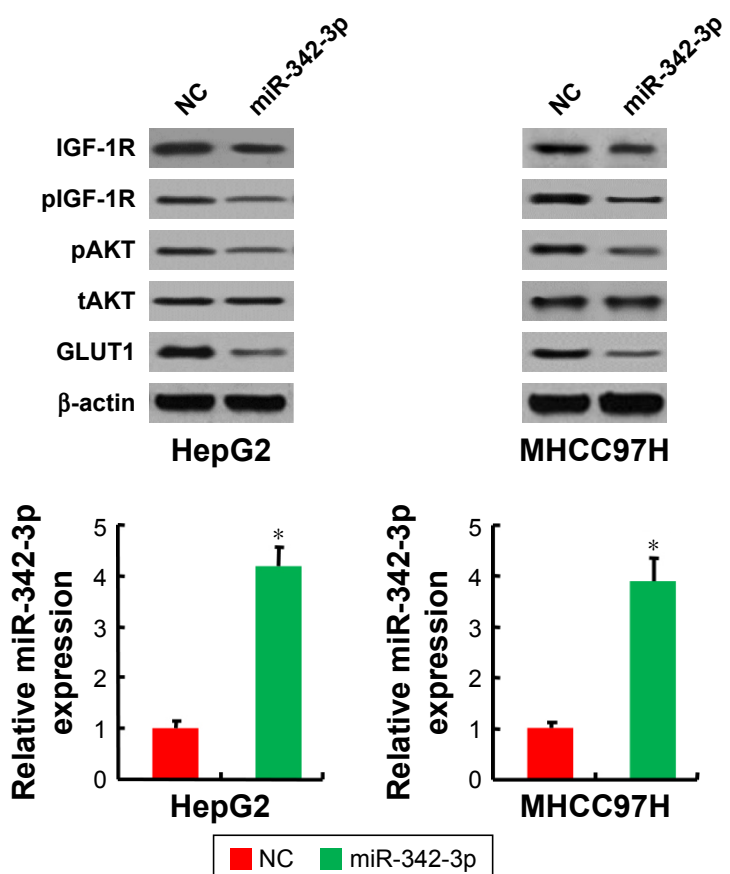

NC miR-342-3p

C
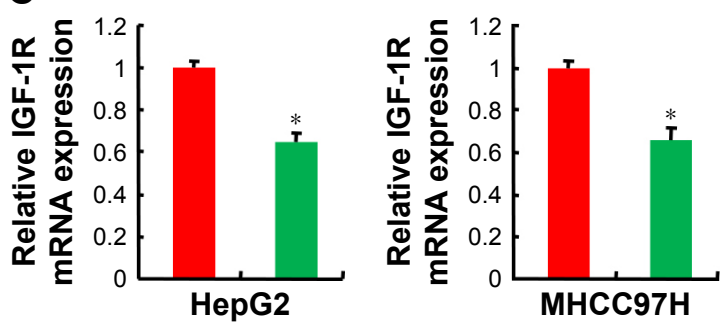

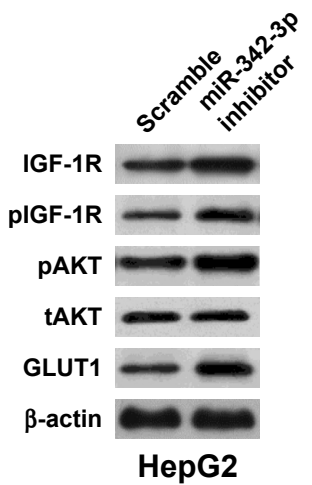



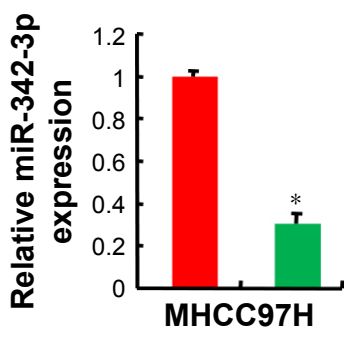

Scramble $\quad$ miR-342-3p inhibitor

D

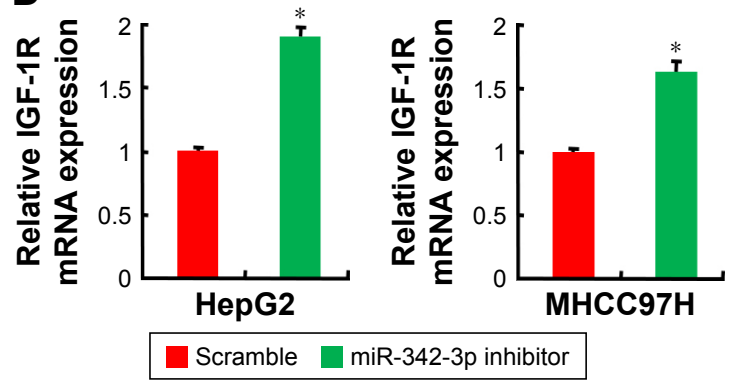

E

Wild-type (WT) IGF-1R 3'-UTR: 5'...GUGUGGGGUGUGUGUGUGUGAGA..3'

hsa-miR-342-3p: 3'-UGCCCACGCUAAAGACACACU CU-5'

Mutated (Mut) IGF-1R 3'-UTR: $\quad 5^{\prime}$...GUGUGGGGUGUGUG GUGUGUCUA...3'
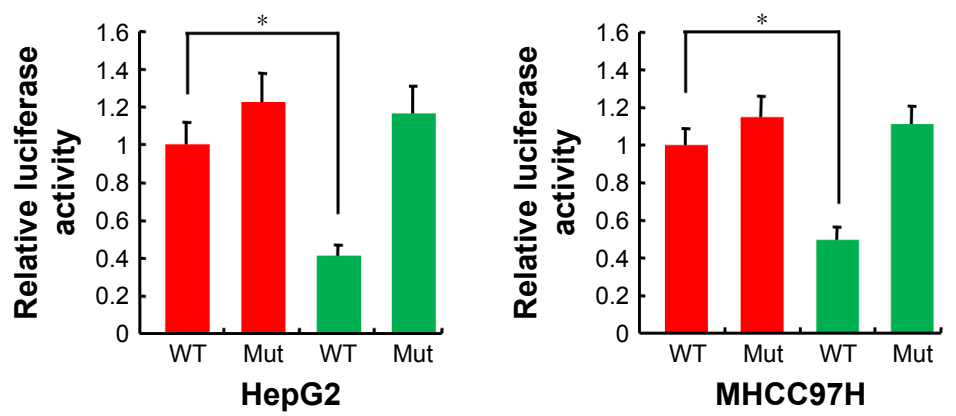

NC miR-342-3p

Figure 2 IGF-IR is a direct target of miR-342-3p.

Notes: (A, B) Immunoblot analysis of HepG2 and MHCC97H cells transfected with NC or miR-342-3p mimics, or scramble or miR-342-3p inhibitor. Scramble was the negative control for miRNA inhibitors. Histograms under the immunoblot graphs indicate corresponding miRNAs expression levels by qRT-PCR. $\beta$-actin was used as a loading control for immunoblot. (C, D) qRT-PCR analysis of IGF-IR mRNA expression levels in the indicated hepatoma cell lines transfected with miR-342-3p mimics or miR-342-3p inhibitor. (E) miRNA luciferase reporter assays of HepG2 and MHCC97H cells transfected with miR-342-3p mimics plus wild-type or mutated IGF-IR reporter. The top panel shows wild-type and mutant forms of putative miR-342-3p target sequences of IGF-IR $3^{\prime}$-UTR. Red font indicates the supposed miR-342-3p binding sites within human IGF-IR $3^{\prime}$-UTR. Red and italicized font indicates the mutations fetched in the IGF-IR $3^{\prime}$-UTR. *P $<0.0$ I.

Abbreviations: NC, negative control; 3'-UTR, 3'-untranslated region. 
by miR-342-3p were dependent on its target IGF-1R. Cell proliferation and colony formation assays determined that IGF-1R knockdown significantly reduced cell proliferation and colony formation ability in hepatoma cells. More importantly, IGF-1R knockdown abolished the ability of miR-342-3p to regulate hepatoma cell proliferation, revealing that miR-342-3p suppresses liver cancer cell proliferation through suppression of IGF-1R expression (Figure 3A

A

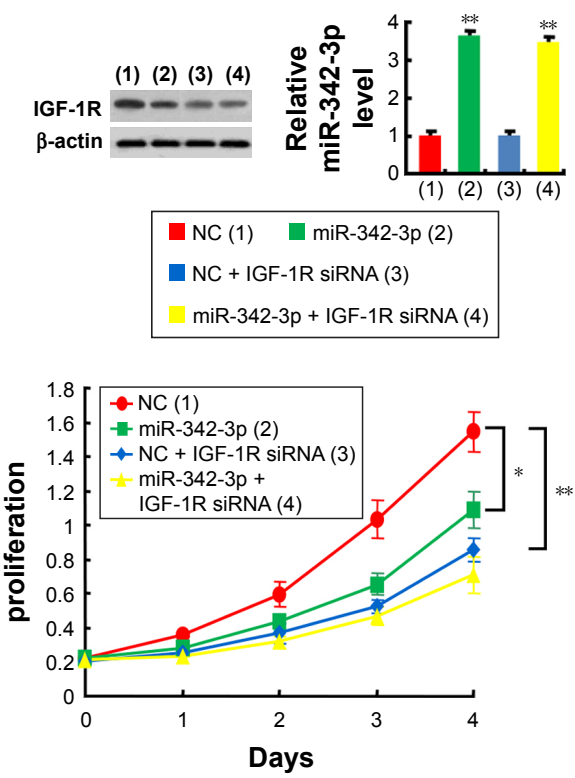

B
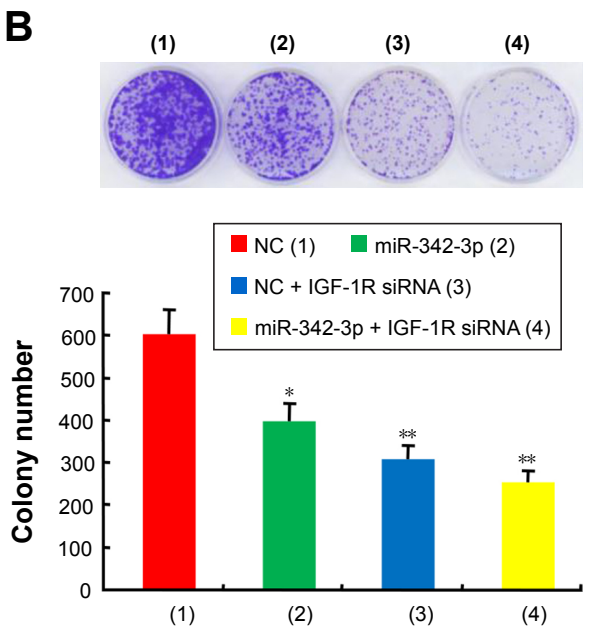

C



Lactate

ATP

(1) (2) (3) (4)



Figure 3 miR-342-3p suppresses hepatoma cell proliferation and glycolysis mainly through inhibition of IGF-IR expression.

Notes: (A) HepG2 cells transfected with NC or miR-342-3p mimics plus IGF-IR siRNA or not. The proliferation of the cells was determined by CCK-8 assay. The typical immunoblot indicates IGF-IR expression. Histograms indicate miR-342-3p expression level determined by qRT-PCR. (B) Colony formation assay of HepG2 cells were transfected as in (A). Typical images show colonies in $35 \mathrm{~mm}$ plates (upper panels). Histograms show colony number. (C-E) HepG2 cells were transfected as mentioned above, glucose uptake, the production of lactate and ATP, ECAR, and OCR were then determined. Representative immunoblot reveals the expression of IGF-IR. qRT-PCR analysis shows miR-342-3p expression. $* P<0.05$, $* * P<0.01$.

Abbreviations: NC, negative control; CCK-8, cell counting kit-8; ECAR, extracellular acidification rate; OCR, oxygen consumption rate; FCCP, P-trifluoromethoxy carbonyl cyanide phenylhydrrazone; Rote/AA, rotenone plus the mitochondrial complex III inhibitor antimycin A. 
and B). To further validate this, we performed IGF-1R rescue experiment. Transfection of miR-342-3p mimics decreased the proliferation of HepG2 cells. These effects were reversed by IGF-1R reexpression in the miR-342-3ptransfected cell lines (Figure S2A). Next, we showed that miR-342-3p mimics decreased glucose uptake, lactate production and ATP generation; however, these effects were eliminated by IGF-1R knockdown. Again, miR-342-3p mimics decreased glucose uptake, lactate production and ATP generation, which could be reversed by IGF-1R reexpression in the miR-342-3p-transfected cells (Figure S2B). miR-342-3p mimics also exhibited decreased ECAR, which reflects overall glycolytic flux, and increased OCR, an indicator of mitochondrial respiration. When IGF-1R was knockdown, miR-342-3p mimics had no effects on the glycolytic phenotype (Figure $3 \mathrm{C}-\mathrm{E}$ ), indicating that miR-342-3p represses the glycolytic phenotype via IGF-1R. Taken together, these results suggest that miR-342-3p restrain glycolysis via inhibition of IGF-1R expression in hepatoma cells.

\section{miR-342-3p inhibits tumor growth and glucose uptake of $\mathrm{HCC}$ in nude mice}

To examine the effects of the miR-342-3p on glycolysis in vivo, we used ${ }^{18} \mathrm{~F}$-FDG microPET scans to measure glucose uptake in tumor xenografts in nude mice. The tumors with miR-342-3p overexpression revealed decreased glucose uptake (Figures 4A and S3). As expected, the tumors with miR-342-3p grew more slowly than the empty control vector. Lactate production analysis of the tumor masses further confirmed that miR-342-3p repressed the lactate production (Figure 4B). In addition, the expression of pAKT, GLUT1, pIGF-1R, IGF-1R, was downregulated by miR-342-3p overexpression, suggesting that the miR-342-3p inhibits tumor growth and glucose uptake in HCC (Figure 4C).

\section{Discussion}

We have demonstrated for the first time, to the best of our knowledge, that miR-342-3p regulates Warburg effect in suppression of liver tumor growth both in vitro and
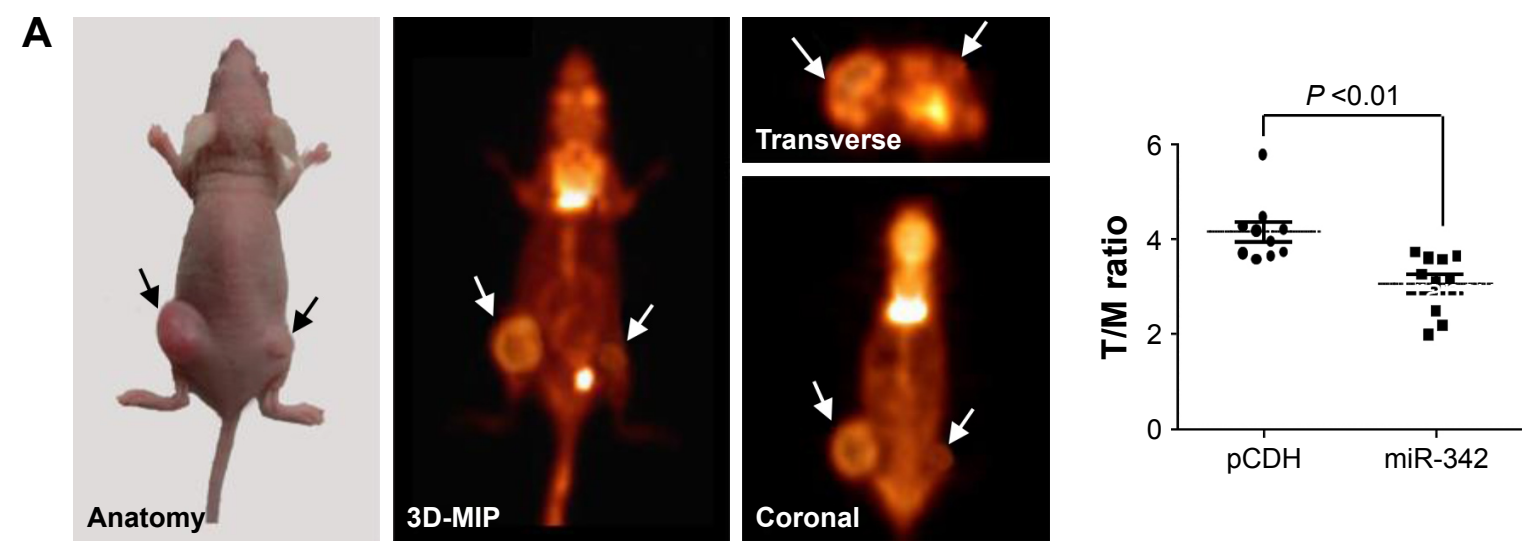

B



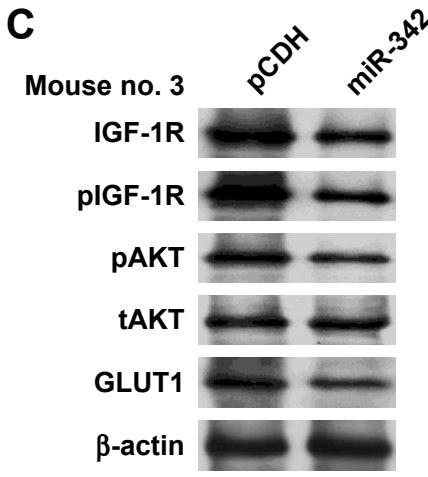

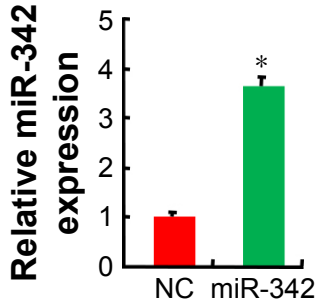

Figure 4 miR-342-3p/IGFIR axis regulates glycolysis and tumor growth in vivo.

Notes: (A) Representative FDG microPET images of living nude mice injected with pCDH or pCDH-miR-342 stably expressing HepG2 cells as indicated. Arrows indicate the tumor glucose uptake. Scatter diagram showed the radioactivity of each ablated tumors. (B) Lactate level of representative tumor tissues from (A). (C) Representative immunoblot and qRT-PCR analysis of the expression of the indicated proteins and miR-342 in representative excised tumor from (A). The indicated tumor tissue from a single mouse in each group was cut into several pieces and then used for immunoblot and qRT-PCR. *P $<0.01$.

Abbreviations: FDG microPET, fluorodeoxyglucose micropositron emission tomography; T/M, tumor to muscle; NC, negative control. 
in vivo. First, glycolytic inhibitor 2-DG weakens the effect of miR-342-3p to regulate hepatoma cell proliferation. Mechanistically, through targeting IGF-1R 3'-UTR directly, miR-342-3p decreases IGF-1R expression, and then decreases the level of pAKT and GLUT1. miR-342-3p inhibits glucose uptake, lactate production, ATP production and induces a switch from glycolysis to mitochondrial respiration through inhibition of IGF-1R mediated the Warburg effect. In vivo micro-PET imaging demonstrates that miR-342-3p significantly inhibits the glucose uptake of liver tumor and lactate production. These observations collectively suggest that the effect of miR-342-3p on the Warburg effect is crucial for regulating $\mathrm{HCC}$ tumorigenesis.

Cancer cells frequently exhibit high level of aerobic glycolysis (the Warburg effect), regarded as a hallmark of cancer. ${ }^{8,9}$ This metabolic reprogramming gives cancer cells a growth benefit by providing energy for cancer cell progression. Targeting glucose metabolism may offer a selective molecular mechanism by which to kill cancer cells. ${ }^{23}$ In solid tumors, miR-342-3p has been identified as a tumor suppressor that inhibits tumorigenesis and cancer progression. For example, miR-342-3p suppresses cervical cancer proliferation, migration and invasion by inhibiting FOXM1. ${ }^{24}$ miR-342-3p targets AGR2 and RAP2B in lung cancer to restrain cancer cell proliferation, migration and invasion. ${ }^{25,26}$ Tai et al reported that miR-342-3p regulates MYC transcriptional activity through direct repression of E2F1 in human lung cancer. ${ }^{27}$ In liver cancer, Zhao et al showed that miR-342-3p affects cell proliferation via regulating NF-kB pathway. ${ }^{22}$ However, whether miR-342-3p regulates the Warburg effect is unknown. We proved that miR-342-3p not only inhibits the Warburg effect but also suppresses hepatoma cell proliferation through inhibition of IGF-1R-mediated Warburg effect.

IGF-1R is a transmembrane protein of the insulin receptor family containing two extracellular $\alpha$ subunits with the ligand-binding site and two transmembrane $\beta$ subunits with intracellular tyrosine kinase activity. It has been demonstrated that IGF-1R was upregulated in numerous cancers, including $\mathrm{HCC}$, renal cell carcinoma, gastric cancer and so on. ${ }^{28-30}$ Deletion of the $I G F-1 R$ gene results in a $50 \%$ decrease in the size of mouse embryos. ${ }^{31}$ IGF-1R interacts with its ligand IGF-1 and plays crucial role in tumorigenesis, proliferation and metastasis through multiple signaling pathways downstream including PI3K/AKT and MAPK/ERK signal pathways. ${ }^{32,33}$ It has been reported that activation of IGF-1R stimulates PI3K/AKT pathway, regulating cell proliferation, migration, invasion. ${ }^{34-37}$ Interference with the function of IGF-1R appears to be potential therapeutic strategy for cancer. In fact, development of drugs targeting IGF-1R is being investigated clinical trials as anticancer treatment. ${ }^{38}$

AKT is a serine/threonine kinase, which is also called protein kinase B. The activity of AKT relays on the phosphorylation at its hydrophobic motif (Ser473) and activation loop (Thr308), which can be activated by variety of growth factors such as IGF. ${ }^{39}$ Phosphorylated AKT (pAKT) is the active form of AKT influencing a diverse range of intracellular functions. ${ }^{40}$ Zhang et al reported that AKT kinase was upregulated and significantly associated with tumor aggressiveness and poor prognosis in patients with HCC. ${ }^{41}$ In addition, previous investigations indicated that increased expression of pAKT was associated with poor prognosis in patients with $\mathrm{HCC}$, colorectal cancer, breast cancer, renal cell carcinoma, etc. ${ }^{42-45}$ Inhibition of AKT signal pathway is a target for cancer therapy. Some researchers reported that the $\mathrm{PI} 3 \mathrm{~K} / \mathrm{AKT}$ pathway was involved in the control of GLUT1 trafficking and activity. ${ }^{21,46,47}$ Inactivation of PI3K/AKT signaling pathway results in GLUT1 expression inhibition, thus affecting glucose metabolism, and finally suppressing the proliferation of colorectal cancer cells. ${ }^{48}$

In short, our data establish molecular mechanism of miR-342-3p in regulating IGF-1R-mediated Warburg effect. miR-342-3p activation may be effective for HCC therapy with IGF-1R overexpression.

\section{Conclusion}

Our investigation demonstrated that miR-342-3p inhibits hepatoma cell proliferation by targeting IGF-1R directly, dampening glycolysis mediated by PI3K/AKT/GLUT1 axis. These findings outline the importance of the miR-342-3p in the Warburg effect and HCC tumorigenesis. Therefore, upregulation of miR-342-3p may be a prospective way to treat IGF-1R overexpression liver cancer patients.

\section{Acknowledgments}

This work was supported by National Natural Science Foundation (81472589, 81672602, 81372834, 81471466, 81572597, 81641023, and 81502264), Logistics Scientific Research project (BWS16J010), Beijing Nova Program (Z171100001117024), and Beijing Capital Special Development Application Program (Z141107002514159).

\section{Disclosure}

The authors report no conflicts of interest in this work.

\section{References}

1. Farazi PA, DePinho RA. Hepatocellular carcinoma pathogenesis: from genes to environment. Nat Rev Cancer. 2006;6(9):674-687.

2. Cabrera R, Nelson DR. Review article: the management of hepatocellular carcinoma. Aliment Pharmacol Ther. 2010;31(4):461-476. 
3. Forner A, Reig M, Bruix J. Alpha-fetoprotein for hepatocellular carcinoma diagnosis: the demise of a brilliant star. Gastroenterology. 2009; 137(1):26-29.

4. Bruix J, Sherman M. Management of hepatocellular carcinoma. Hepatology. 2005;42(5):1208-1236.

5. Llovet JM, Burroughs A, Bruix J. Hepatocellular carcinoma. Lancet. 2003;362(9399):1907-1917.

6. Marrero JA, Welling T. Modern diagnosis and management of hepatocellular carcinoma. Clin Liver Dis. 2009;13(2):233-247.

7. Jemal A, Bray F, Center MM, Ferlay J, Ward E, Forman D. Global cancer statistics. CA Cancer J Clin. 2011;61(2):69-90.

8. Hanahan D, Weinberg RA. Hallmarks of cancer: the next generation. Cell. 2011;144(5):646-674.

9. Vander Heiden MG, Cantley LC, Thompson CB. Understanding the Warburg effect: the metabolic requirements of cell proliferation. Science. 2009;324(5930):1029-1033.

10. Bartel DP. MicroRNAs: genomics, biogenesis, mechanism, and function. Cell. 2004;116(2):281-297.

11. Calin GA, Croce CM. MicroRNA signatures in human cancers. Nat Rev Cancer. 2006;6(11):857-866.

12. Gaur A, Jewell DA, Liang Y, et al. Characterization of microRNA expression levels and their biological correlates in human cancer cell lines. Cancer Res. 2007;67(6):2456-2468.

13. Srivastava SK, Ahmad A, Zubair H, et al. MicroRNAs in gynecological cancers: small molecules with big implications. Cancer Lett. 2017;407:123-138.

14. Wang Q, Li PC, Li AL, et al. Plasma specific miRNAs as predictive biomarkers for diagnosis and prognosis of glioma. J Exp Clin Cancer Res. 2012;31:97.

15. Tao K, Yang J, Guo Z, Hu Y, Sheng H, Gao H, Yu H. Prognostic value of miR-221-3p, miR-342-3p and miR-491-5p expression in colon cancer. Am J Transl Res. 2014;6(4):391-401.

16. Jung CK, Jung SH, Yim SH, et al. Predictive microRNAs for lymph node metastasis in endoscopically resectable submucosal colorectal cancer. Oncotarget. 2016;7(22):32902-32915.

17. Lee KH, Lee JK, Choi DW, et al. Postoperative prognosis prediction of pancreatic cancer with seven microRNAs. Pancreas. 2015;44(5): 764-768.

18. Zhang S, Liu L, Lv Z, Li Q, Gong W, Wu H. MicroRNA-342-3p inhibits the proliferation, migration, and invasion of osteosarcoma cells by targeting astrocyte-elevated gene-1 (AEG-1). Oncol Res. 2017;25(9): 1505-1515.

19. Wang SH, Ma F, Tang ZH. Long non-coding RNA H19 regulates FOXM1 expression by competitively binding endogenous miR-342-3p in gallbladder cancer. J Exp Clin Cancer Res. 2016;35(1):160.

20. Pollak MN. Insulin and insulin-like growth factor signalling in neoplasia. Nat Rev Cancer. 2008;8(12):915-928.

21. Phadngam S, Castiglioni A, Ferraresi A, Morani F, Follo C, Isidoro C. PTEN dephosphorylates AKT to prevent the expression of GLUT1 on plasmamembrane and to limit glucose consumption in cancer cells. Oncotarget. 2016;7(51):84999-85020.

22. Zhao L, Zhang YB. miR-342-3p affects hepatocellular carcinoma cell proliferation via regulating NF-kB pathway. Biochem Biophys Res Commun. 2015;457(3):370-377.

23. Hamanaka RB, Chandel NS. Targeting glucose metabolism for cancer therapy. J Exp Med. 2012;209(2):211-215.

24. Li XR, Chu HJ, Lv T, Wang L, Kong SF, Dai SZ. miR-342-3p suppresses proliferation, migration and invasion by targeting FOXM1 in human cervical cancer. FEBS Lett. 2014;588(17):3298-3307.

25. Xue XF, Fei XY, Hou WJ, Zhang YJ, Liu L, Hu RK. miR-342-3p suppresses cell proliferation and migration by targeting AGR2 in non-small cell lung cancer. Cancer Lett. 2018;412:170-178.

26. Xie X, Liu HT, Wang MS, et al. miR-342-3p targets RAP2B to suppress proliferation and invasion of non-small cell lung cancer cells. Tumour Biol. 2015;36(7):5031-5038.

27. Tai MC, Kajino T, Nakatochi M, et al. miR-342-3p regulates MYC transcriptional activity via direct repression of E2F1 in human lung cancer. Carcinogenesis. 2015;36(12):1464-1473.
28. E C, Li J, Shao D, et al. The insulin-like growth factor-I receptor inhibitor picropodophyllin-induced selective apoptosis of hepatocellular carcinoma cell through a caspase-dependent mitochondrial pathway. Oncol Res. 2013;21(2):103-110.

29. Sichani MM, Yazdi FS, Moghaddam NA, et al. Prognostic value of insulin-like growth factor-I receptor expression in renal cell carcinoma. Saudi J Kidney Dis Transpl. 2010;21(1):69-74.

30. Gryko M, Kiśluk J, Cepowicz D, et al. Expression of insulin-like growth factor receptor type 1 correlate with lymphatic metastases in human gastric cancer. Pol J Pathol. 2014;65(2):135-140.

31. Efstratiadis A. Genetics of mouse growth. Int J Dev Biol. 1998;42(7): 955-976.

32. Cao Z, Liu L-Z, Dixon DA, Zheng JZ, Chandran B, Jiang B-H. Insulinlike growth factor-I induces cyclooxygenase-2 expression via PI3K, MAPK and PKC signaling pathways in human ovarian cancer cells. Cell Signal. 2007;19(7):1542-1553.

33. Guo T, Feng Y, Liu Q, Yang X, Jiang T, Chen Y, Zhang Q. MicroRNA320a suppresses in GBM patients and modulates glioma cell functions by targeting IGF-1R. Tumour Biol. 2014;35(11):11269-11275.

34. Pollak MN. Insulin-like growth factors and neoplasia. Novartis Found Symp. 2004;262:84-98; discussion: 98-107, 265-268.

35. Werner H, LeRoith D. The role of the insulin-like growth factor system in human cancer. Adv Cancer Res. 1996;68:183-223.

36. Pollak M. The insulin and insulin-like growth factor receptor family in neoplasia: an update. Nat Rev Cancer. 2012;12(3):159-169.

37. King H, Aleksic T, Haluska P, Macaulay VM. Can we unlock the potential of IGF-1R inhibition in cancer therapy? Cancer Treat Rev. 2014; 40(9):1096-1105.

38. Hartog H, Wesseling J, Boezen HM, van der Graaf WT. The insulinlike growth factor 1 receptor in cancer: old focus, new future. Eur $J$ Cancer. 2007;43(13):1895-1904.

39. Wang L, Guo W, Ma J, et al. Aberrant SIRT6 expression contributes to melanoma growth: role of the autophagy paradox and IGF-AKT signaling. Autophagy. Epub. 2017 Dec 31.

40. Vivanco I, Sawyers CL. The phosphatidylinositol 3-kinase AKT pathway in human cancer. Nat Rev Cancer. 2002;2(7):489-501.

41. Zhang Y, Guo X, Yang M, Yu L, Li Z, Lin N. Identification of AKT kinases as unfavorable prognostic factors for hepatocellular carcinoma by a combination of expression profile, interaction network analysis and clinical validation. Mol BioSyst. 2014;10(2):215-222.

42. Chen YL, Chen PM, Ming YZ, Lin PY, Chu CP, Chu PY. Phosphorylated AKT expression in tumor-adjacent normal tissue is associated with poor prognosis in patients with hepatocellular carcinoma. Oncol Lett. 2017;14(6):7461-7466.

43. Malinowsky K, Nitsche U, Janssen KP, et al. Activation of the PI3K/ AKT pathway correlates with prognosis in stage II colon cancer. $\mathrm{Br} J$ Cancer. 2014;110(8):2081-2089.

44. Tokunaga E, Kimura Y, Oki E, et al. Akt is frequently activated in HER2/ neu-positive breast cancers and associated with poor prognosis among hormone-treated patients. Int J Cancer. 2006;118(2):284-289.

45. Hager M, Haufe H, Kemmerling R, Hitzl W, Mikuz G, Moser PL, Kolbitsch C. Increased activated Akt expression in renal cell carcinomas and prognosis. J Cell Mol Med. 2009;13(8B):2181-2188.

46. Wieman HL, Wofford JA, Rathmell JC. Cytokine stimulation promotes glucose uptake via phosphatidylinositol-3 kinase/Akt regulation of Glut1 activity and trafficking. Mol Biol Cell. 2007;18(4):1437-1446.

47. Melstrom LG, Salabat MR, Ding XZ, Milam BM, Strouch M, Pelling JC, Bentrem DJ. Apigenin inhibits the GLUT-1 glucose transporter and the phosphoinositide 3-kinase/Akt pathway in human pancreatic cancer cells. Pancreas. 2008;37(4):426-431.

48. Wu XL, Wang LK, Yang DD, et al. Effects of Glut1 gene silencing on proliferation, differentiation, and apoptosis of colorectal cancer cells by targeting the TGF- $\beta$ /PI3K-AKT-mTOR signaling pathway. $J$ Cell Biochem. 2018;119(2):2356-2367. 


\section{Supplementary materials}

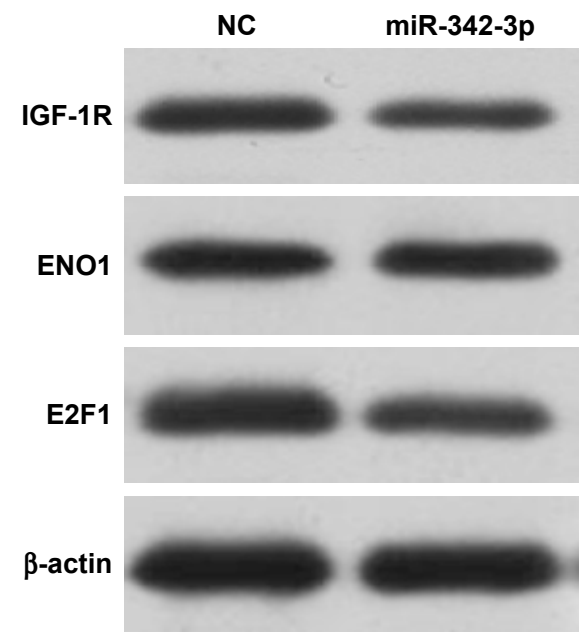

Figure SI Screening for the target of miR-342-3 in HEK293T cells.

Notes: Immunoblot analysis of HEK293T cells transfected with NC or miR-342-3p mimics. $\beta$-actin used as a loading control.

Abbreviation: NC, negative control.
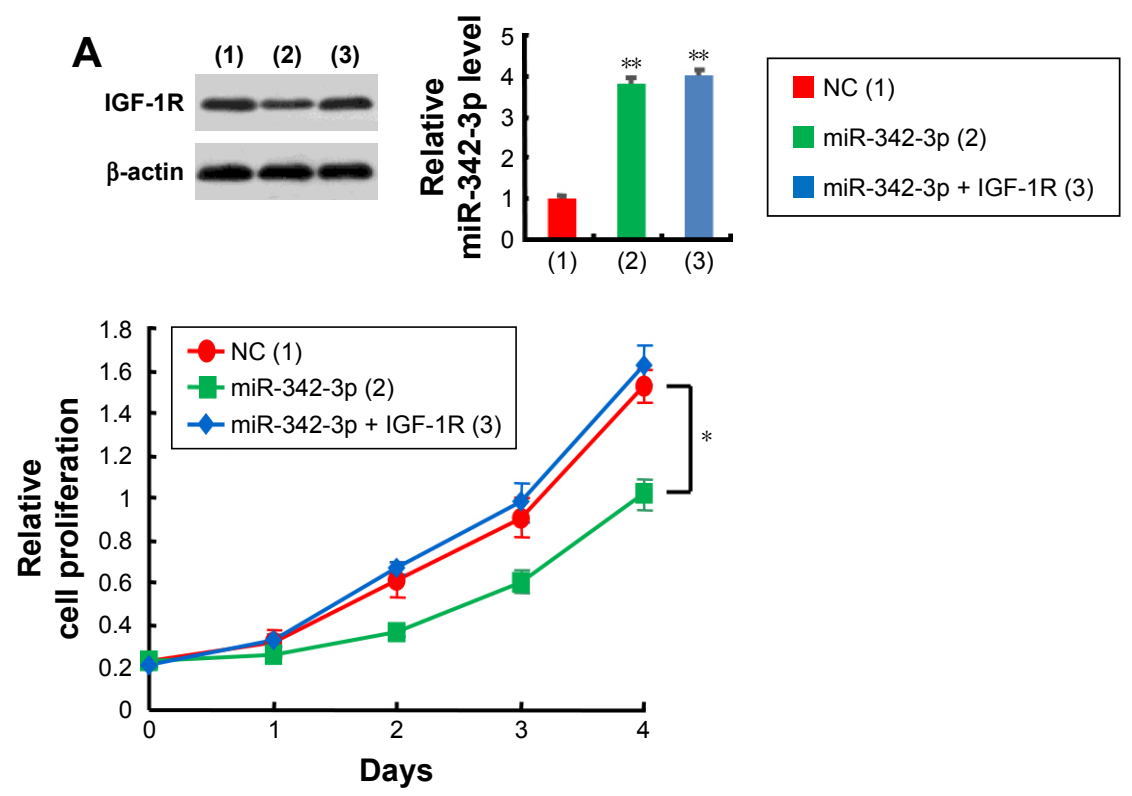

\section{B}
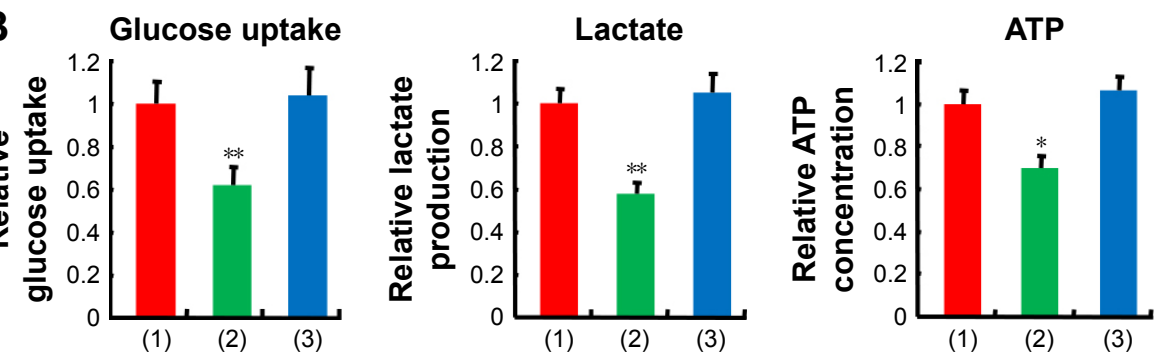

NC (1) $\quad$ miR-342-3p (2) $\quad$ miR-342-3p + IGF-1R (3)

Figure S2 miR-342-3p suppresses proliferation, glucose uptake and the production of lactate and ATP through inhibition of IGF-IR expression in HCC cells.

Notes: (A) HepG2 cells were transfected with miR-342-3p mimics or miR-342-3p mimics plus IGF-IR expression vector. The proliferation of the cells was detected by CCK-8 assay. The representative immunoblot shows IGF-IR expression. Histograms show miR-342-3p expression determined by qRT-PCR. $\beta$-actin was used as a loading control. (B) HepG2 cells were transfected as in (A), glucose uptake and the production of lactate and ATP were determined. $* P<0.05 ; * * P<0.0$ I.

Abbreviations: NC, negative control; CCK-8, cell counting kit-8. 


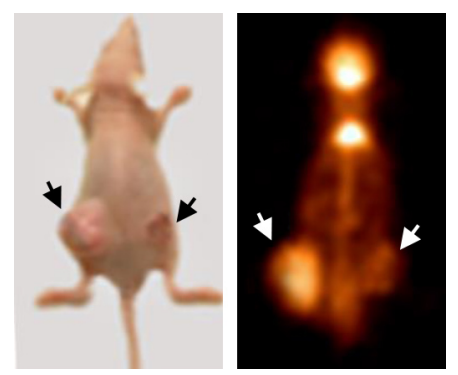

Figure S3 miR-342-3p/IGF-IR axis regulates glycolysis and tumor growth in vivo. Notes: Representative FDG microPET images of living nude mice injected with $\mathrm{pCDH}$ or $\mathrm{pCDH}-\mathrm{miR}-342$ stably expressing HepG2 cells as indicated. Arrows indicate the tumor glucose uptake.

Abbreviation: FDG microPET, fluorodeoxyglucose micropositron emission tomography.

\section{Publish your work in this journal}

OncoTargets and Therapy is an international, peer-reviewed, open access journal focusing on the pathological basis of all cancers, potential targets for therapy and treatment protocols employed to improve the management of cancer patients. The journal also focuses on the impact of management programs and new therapeutic agents and protocols on
Dovepress

patient perspectives such as quality of life, adherence and satisfaction. The manuscript management system is completely online and includes a very quick and fair peer-review system, which is all easy to use. Visit http://www.dovepress.com/testimonials.php to read real quotes from published authors.

Submit your manuscript here: http://www.dovepress.com/oncotargets-and-therapy-journal 